\title{
Expansions for Coulomb Wave Functions
}

\author{
By J. Boersma
}

Abstract. In this paper we derive a number of expansions for Whittaker functions, regular and irregular Coulomb wave functions. The main result consists of a new expansion for the irregular Coulomb wave functions of orders zero and one in terms of regular Coulomb wave functions. The latter expansions are especially useful with a view to the numerical computation of irregular Coulomb wave functions.

1. Introduction. Coulomb wave functions which play an important role in various problems in quantum mechanics (see e.g. Breit's foreword to [7], Messiah [6]) arise as solutions of the differential equation

$$
\frac{d^{2} w}{d \rho^{2}}+\left[1-\frac{2 \eta}{\rho}-\frac{L(L+1)}{\rho^{2}}\right] w=0
$$

where $\eta$ is a real parameter, $L$ a nonnegative integer, and $\rho>0$. The Eq. (1.1) is a special case of Whittaker's equation (see Slater [8, Sections 1.6, 5.3]), hence its solutions can be expressed in terms of Whittaker functions. It is customary to define the regular Coulomb wave function $F_{L}(\eta, \rho)$ and the irregular Coulomb wave function $G_{L}(\eta, \rho)$ in the following way (cf. [8, Formulas (5.3.6), (5.3.7)])

$$
\begin{aligned}
& F_{L}(\eta, \rho)=A M_{i \eta, L+1 / 2}(2 i \rho), \\
& G_{L}(\eta, \rho)=i A M_{i \eta, L+1 / 2}(2 i \rho)+B W_{i \eta, L+1 / 2}(2 i \rho)
\end{aligned}
$$

where

$$
\begin{aligned}
& A=\frac{|\Gamma(L+1+i \eta)| \exp [-\pi \eta / 2-(L+1) \pi i / 2]}{2(2 L+1) !}, \\
& B=\exp \left[\pi \eta / 2+L \pi i / 2-i \sigma_{L}\right]
\end{aligned}
$$

with $\sigma_{L}$ defined through

$$
\exp \left(i \sigma_{L}\right)=\frac{\Gamma(L+1+i \eta)}{|\Gamma(L+1+i \eta)|} .
$$

The Whittaker functions $M_{\kappa, \mu}(z), W_{\kappa, \mu}(z)$ are defined in [8, Sections 1.6, 1.7] (see also (2.3), (2.9)). For a comprehensive collection of results on Coulomb wave functions, the reader is referred to Abramowitz and Stegun [1, Chapter 14].

The plan of the present paper may be outlined as follows. In Section 2 we present a number of expansions in terms of Whittaker functions. By means of (1.2) these expansions are translated into expansions for regular and irregular Coulomb wave functions (Sections 3 and 4). Finally, in Section 5 the computational aspects of the various expansions are discussed.

Received March 14, 1968, revised July 19, 1968. 
2. Expansions for Whittaker Functions. We start from the following expansion quoted from Buchholz $[2, \S 11$, Formula $(16 \beta)]$ :

$$
z^{\mu+1} e^{w z / 2}=\sum_{n=0}^{\infty}(2 n+2 \mu+1) \Gamma(n+2 \mu+1) P_{n}{ }^{(\mu+\kappa, \mu-\kappa)}(w) \frac{M_{\kappa, n+\mu+1 / 2}(z)}{\Gamma(2 n+2 \mu+2)}
$$

valid for $2 \mu \neq-2,-3, \cdots$ and arbitrary (complex) $w, z$ and $\kappa$. The notation $P_{n}{ }^{(\alpha, \beta)}(w)$ stands for the Jacobi polynomial of degree $n$ (see [3, Section 10.8]). In (2.1) we substitute $w=-1$ and $\mu=m$ where $m$ is a nonnegative integer; then the Jacobi polynomial can be evaluated by means of [3, Formulas 10.8(3), (13)] and we obtain

$$
\begin{array}{r}
z^{m+1} e^{-z / 2}=\sum_{n=0}^{\infty} \frac{(n+2 m) !(m+1-\kappa)_{n}}{(2 n+2 m) ! n !}(-1)^{n} M_{\kappa, n+m+1 / 2}(z), \\
m=0,1,2, \cdots .
\end{array}
$$

The present result is basic in that any power series made up of terms $z^{m+1} e^{-z / 2}$ can be rearranged (by means of (2.2)) to an expansion in terms of Whittaker functions $M_{\kappa, n+1 / 2}(z)(n=0,1,2, \cdots)$. In the remainder of this section we shall derive such expansions for the functions $e^{-z / 2}, z(d / d z) M_{\kappa, m+1 / 2}(z)$, and $W_{\kappa, 1 / 2}(z)$. In deriving these expansions we make use of the following power series representation for $M_{\kappa, \mu}(z)$,

$$
M_{\kappa, \mu}(z)=z^{\mu+1 / 2} e^{-z / 2} \sum_{r=0}^{\infty} \frac{\left(\mu+\frac{1}{2}-\kappa\right)_{r}}{(2 \mu+1)_{r} r !} z^{r}
$$

valid for $2 \mu \neq-1,-2, \cdots$ (cf. [8, Formula (1.6.4)]).

We first derive an expansion for the function $e^{-z / 2}$ (this expansion is needed later on in connection with the term $e^{-z / 2}$ occurring in the representation (2.9) for the Whittaker function $\left.W_{\kappa, 1 / 2}(z)\right)$. For that purpose we start from (2.1) with $w=-1$ and take the limit for $\mu \rightarrow-1$. The undesirable term $M_{\kappa,-1 / 2}(z)$ is removed by applying the recurrence relation

$$
\begin{aligned}
M_{\kappa, \mu-1}(z) & +\left[\frac{2 \kappa}{(2 \mu-1)(2 \mu+1)}-\frac{1}{z}\right] M_{\kappa, \mu}(z) \\
& -\frac{(2 \mu+1-2 \kappa)(2 \mu+1+2 \kappa)}{8 \mu(2 \mu+1)^{2}(2 \mu+2)} M_{\kappa, \mu+1}(z)=0 .
\end{aligned}
$$

The latter relation follows by suitably combining [8, Formulas (2.5.1), (2.5.6)]. As our final result we obtain the expansion

$$
\begin{aligned}
e^{-z / 2}= & \frac{M_{\kappa, 1 / 2}(z)}{z}-\frac{1-\kappa}{2} M_{\kappa, 1 / 2}(z)+\frac{(1-\kappa)(1+\kappa)}{12} M_{\kappa, 3 / 2}(z) \\
& +\kappa \sum_{n=1}^{\infty} \frac{(1-\kappa)_{n}}{(2 n) ! n(n+1)}(-1)^{n} M_{\kappa, n+1 / 2}(z) .
\end{aligned}
$$

We remark that the present expansion, which is of slightly different type than (2.1), (2.2), is to be considered as the consistent extension of (2.2) to the case $m=-1$. In principle, similar extensions might be stated if $m$ is an arbitrary negative integer. However, the resulting expansions being rather complicated we will not pursue this matter. 
Next, we present an expansion for the derivative of $M_{\kappa, m+1 / 2}(z)(m=0,1$, $2, \cdots)$. According to (2.2), (2.3) we have

where

$$
z^{-m+1} \frac{d}{d z}\left[z^{m} M_{\kappa, m+1 / 2}(z)\right]=m M_{\kappa, m+1 / 2}(z)+z(d / d z) M_{\kappa, m+1 / 2}(z)
$$

$$
\begin{aligned}
& =\left(m+\frac{1}{2}\right) e^{-z / 2} \sum_{r=0}^{\infty} \frac{(m+1-\kappa)_{r}(r+2 m-2 \kappa)}{(2 m+1)_{r} r !(r+m-\kappa)} z^{r+m+1} \\
& =\left(m+\frac{1}{2}\right) \sum_{p=0}^{\infty} \frac{(m+1-\kappa)_{p}}{(2 p+2 m) !}(-1)^{p} S_{p} M_{\kappa, p+m+1 / 2}(z)
\end{aligned}
$$

$$
S_{p}=\sum_{r=0}^{p} \frac{(r+p+2 m) !}{(2 m+1)_{r}(p-r) ! r !}(-1)^{r} \frac{r+2 m-2 \kappa}{r+m-\kappa} .
$$

In the appendix, $S_{p}$ is evaluated in closed form (see (A.2)) and we obtain

$$
\begin{aligned}
& z(d / d z) M_{\kappa, m+1 / 2}(z)=(m+1) M_{\kappa, m+1 / 2}(z)+\frac{(2 m+1) !}{2} \\
& \quad \times \sum_{n=1}^{\infty} \frac{(-1)^{n}}{(2 n+2 m) !}\left[(m+1+\kappa)_{n}+(-1)^{n}(m+1-\kappa)_{n}\right] M_{\kappa, n+m+1 / 2}(z)
\end{aligned}
$$

valid for $m=0,1,2, \cdots$.

In order to expand the Whittaker function $W_{k, 1 / 2}(z)$ we start from the power series representation (cf. [8, Formula (1.7.18)])

$$
\begin{aligned}
W_{\kappa, 1 / 2}(z)=\frac{1}{\Gamma(-\kappa)} & {\left[M_{\kappa, 1 / 2}(z) \log z-\kappa^{-1} e^{-z / 2}+z e^{-z / 2}\right.} \\
& \left.\times \sum_{r=0}^{\infty} \frac{(1-\kappa)_{r}}{(r+1) ! r !}\{\psi(r+1-\kappa)-\psi(r+1)-\psi(r+2)\} z^{r}\right]
\end{aligned}
$$

where $\psi(w)$ is the common notation for the logarithmic derivative of the gamma function. The term $e^{-z / 2}$ in (2.9) is replaced by its expansion (2.5). Further, let the infinite series term in (2.9) be denoted by $H(z)$ then using (2.2) $H(z)$ can be rearranged to a series in terms of Whittaker functions, viz.

$$
H(z)=\sum_{p=0}^{\infty} \frac{(1-\kappa)_{p}}{(2 p) !}(-1)^{p}\left\{T_{p}(1-\kappa)-T_{p}(1)-T_{p}(2)\right\} M_{\kappa, p+1 / 2}(z)
$$

where we introduced the notation

$$
T_{p}(a)=\sum_{r=0}^{p} \frac{(r+p) !}{(r+1) !(p-r) ! r !}(-1)^{r} \psi(r+a) .
$$

A closed-form result for $T_{p}(a)$ is obtained in the appendix, Formulas (A.4), (A.7). As our final result we present the expansion

$$
\begin{aligned}
& W_{\kappa, 1 / 2}(z)=\frac{1}{\Gamma(1-\kappa)} \\
& \times\left[\left\{z^{-1}-\kappa[\log z+\psi(1-\kappa)+2 \gamma-1]-(1-\kappa) / 2\right\} M_{\kappa, 1 / 2}(z)\right. \\
& \begin{array}{l}
+\frac{(1-\kappa)(1+\kappa)}{12} M_{\kappa, 3 / 2}(z) \\
\left.+\kappa \sum_{n=1}^{\infty} \frac{(-1)^{n}}{(2 n) ! n(n+1)}\left[(1+\kappa)_{n}+(-1)^{n}(1-\kappa)_{n}\right] M_{\kappa, n+1 / 2}(z)\right] .
\end{array}
\end{aligned}
$$


Notice that in (2.12) we introduced the known values $\psi(1)=-\gamma, \psi(2)=-\gamma+1$ with $\gamma$ denoting Euler's constant (cf. [3, Formulas 1.7(4), (9)]).

As a generalization of (2.12) one might derive a similar expansion for the Whittaker function $W_{\kappa, m+1 / 2}(z)$ if $m$ is a positive integer. However, the power series representation of the latter function will now contain terms $z^{-r} e^{-z / 2}$ with $r=0$, $1, \cdots, m$ (cf. [8, Formula (1.7.18)]). As we did not consider the expansion of such terms in a series of Whittaker functions $M_{\kappa, n+1 / 2}(z)$ (see the remark following (2.5)) we will abandon this generalization.

The series of Whittaker functions $M_{\kappa, n+1 / 2}(z)$ as presented in this section may be called series of the Neumann type, because of their analogy to the Neumann series from the theory of Bessel functions (see Watson [11, Chapter 16]). The expansion (2.12), for example, has as its counterpart the following expansion of $Y_{0}(z)$ in terms of Bessel functions $J_{n}(z)$,

$$
Y_{0}(z)=\frac{2}{\pi}\left[\{\log (z / 2)+\gamma\} J_{0}(z)+2 \sum_{n=1}^{\infty} \frac{(-1)^{n-1}}{n} J_{2 n}(z)\right]
$$

(cf. [11, Section 3.57]). Some further results on Neumann type series of Whittaker functions are collected in $[2, \S 11.3]$.

3. Expansions for Regular Coulomb Wave Functions. By means of (1.2) part of the expansions of Section 2 will be translated into expansions in terms of regular Coulomb wave functions. In the process of translation we shall need the following identities for the gamma and $\psi$ functions:

$$
\frac{e^{\pi \eta / 2}}{|\Gamma(1+i \eta)|}=\left[\frac{e^{\pi \eta}}{\Gamma(1+i \eta) \Gamma(1-i \eta)}\right]^{1 / 2}=\left[\frac{e^{\pi \eta} \sinh \pi \eta}{\pi \eta}\right]^{1 / 2}=\left[\frac{e^{2 \pi \eta}-1}{2 \pi \eta}\right]^{1 / 2}
$$

(cf. [3, Formulas 1.2(1), (6)])

$$
\begin{aligned}
\operatorname{Im} \psi(1+i \eta) & =(1 / 2 i)[\psi(1+i \eta)-\psi(1-i \eta)] \\
& =-1 / 2 \eta+\frac{\pi}{2} \operatorname{coth} \pi \eta=-1 / 2 \eta+\frac{\pi}{2} \frac{e^{2 \pi \eta}+1}{e^{2 \pi \eta}-1}
\end{aligned}
$$

(cf. [3, Formula 1.7(11)]) where Im $w$ denotes the imaginary part of $w$. Further, the following notation will turn out to be quite useful in the subsequent analysis,

$$
\delta_{m, L}=\sigma_{m+L}-\sigma_{m}+L \pi / 2, \quad m, L=0,1,2, \cdots,
$$

where $\sigma_{L}$ is given by (1.4). Hence, the definition (3.3) is equivalent to

$$
\delta_{m, L}=\sum_{n=1}^{L} \arctan [\eta /(m+n)]+L \pi / 2 .
$$
obtain

We first consider the expansion (2.1). Substituting $\mu=0, \kappa=i \eta, z=2 i \rho$ we

$$
\rho e^{i w \rho}=\left[\frac{e^{2 \pi \eta}-1}{2 \pi \eta}\right]^{1 / 2} \sum_{L=0}^{\infty}(2 L+1) \frac{L !}{\left|(1+i \eta)_{L}\right|} i^{L} P_{L}^{(i \eta,-i \eta)}(w) F_{L}(\eta, \rho)
$$

valid for arbitrary complex $w$. After a similar substitution the expansion (2.2) reduces to 


$$
\begin{aligned}
\rho^{m+1} e^{-i p}= & {\left[\frac{e^{2 \pi \eta}-1}{2 \pi \eta}\right]^{1 / 2} \frac{2^{-m}}{\left|(1+i \eta)_{m}\right|} } \\
& \times \sum_{L=0}^{\infty}(2 L+2 m+1) \frac{(L+2 m) !}{L !} \exp \left(-i \delta_{m, L}\right) F_{L+m}(\eta, \rho)
\end{aligned}
$$

valid for $m=0,1,2, \cdots$. A special case of (3.6) viz. the case $m=0$, was attributed in [9] to Henrici.

Finally, from (2.8) we deduce the following expansion for the derivative of a regular Coulomb wave function

$$
\begin{aligned}
\rho(d / d \rho) F_{L}(\eta, \rho)= & (L+1) F_{L}(\eta, \rho) \\
& +\sum_{n=1}^{\infty}(2 n+2 L+1)(-1)^{n} \cos \delta_{L, n} F_{L+n}(\eta, \rho)
\end{aligned}
$$

valid for $L=0,1,2, \cdots$.

4. Expansions for $G_{0}(\eta, \rho), G_{1}(\eta, \rho)$. In the present section we will derive expansions for $G_{0}(\eta, \rho), G_{1}(\eta, \rho)$ in terms of regular Coulomb wave functions. According to (1.2) $G_{0}(\eta, \rho)$ is given by

$$
G_{0}(\eta, \rho)=i F_{0}(\eta, \rho)+\exp \left[\pi \eta / 2-i \sigma_{0}\right] W_{i \eta, 1 / 2}(2 i \rho) .
$$

The Whittaker function $W_{i \eta, 1 / 2}(2 i \rho)$ is now replaced by the expansion (2.12) with $\kappa=i \eta, z=2 i \rho$. By means of (1.2) the latter expansion can again be expressed in terms of regular Coulomb wave functions. The resulting expansion for $G_{0}(\eta, \rho)$ can be simplified further by an application of (3.1), (3.2) and we ultimately obtain

$$
\begin{aligned}
G_{0}(\eta, \rho)=\frac{e^{2 \pi \eta}-1}{2 \pi \eta}[ & \left\{\rho^{-1}+2 \eta[\log 2 \rho+\operatorname{Re} \psi(1+i \eta)+2 \gamma-3 / 2]\right\} F_{0}(\eta, \rho) \\
& -\left(1+\eta^{2}\right)^{1 / 2} F_{1}(\eta, \rho) \\
& \left.-4 \eta \sum_{L=1}^{\infty} \frac{2 L+1}{L(L+1)}(-1)^{L} \cos \delta_{0, L} F_{L}(\eta, \rho)\right]
\end{aligned}
$$

where $\delta_{0, L}$ is defined by (3.3).

The present expansion has been checked by substituting the right-hand side of (4.2) in the differential equation (1.1) with $L=0$. Using (3.7), (4.4) with $L=0$ it has been shown that the expansion (4.2) is indeed a solution of (1.1). Further, it can be verified that (4.2) shows the proper behaviour near $\rho=0$. Hence, the expansion (4.2) is correct.

As an obvious generalization of (4.2) one might consider expansion of the function $G_{L}(\eta, \rho)(L=1,2, \cdots)$ in a series of regular Coulomb wave functions. However, in order to make such a generalization one first has to extend (2.12) to an expansion for the Whittaker function $W_{\kappa, L+1 / 2}(z)$. Therefore, in the following we will use a different approach. At the same time we confine our investigation to the function $G_{1}(\eta, \rho)$.

From [1, Formulas 14.2.1, 2, 3] we quote the following recurrence relations for the Coulomb wave functions:

$$
\begin{aligned}
L d u_{L} / d \rho & =\left(L^{2}+\eta^{2}\right)^{1 / 2} u_{L-1}-\left(L^{2} / \rho+\eta\right) u_{L}, \\
(L+1) d u_{L} / d \rho & =\left[(L+1)^{2} / \rho+\eta\right] u_{L}-\left[(L+1)^{2}+\eta^{2}\right]^{1 / 2} u_{L+1},
\end{aligned}
$$




$$
\begin{aligned}
L\left[(L+1)^{2}+\eta^{2}\right]^{1 / 2} u_{L+1}= & (2 L+1)[\eta+L(L+1) / \rho] u_{L} \\
& -(L+1)\left(L^{2}+\eta^{2}\right)^{1 / 2} u_{L-1}
\end{aligned}
$$

where $u_{L}$ stands for either $F_{L}(\eta, \rho)$ or $G_{L}(\eta, \rho)$. As a special case of (4.4) we have

$$
D F_{0}(\eta, \rho) \equiv[-d / d \rho+1 / \rho+\eta] F_{0}(\eta, \rho)=\left(1+\eta^{2}\right)^{1 / 2} F_{1}(\eta, \rho)
$$

while the same relation holds if $F$ is replaced by $G$. Now we apply the operator $D$ (as defined by (4.6)) to both sides of (4.2), obtaining

$$
\begin{aligned}
& \left(1+\eta^{2}\right)^{1 / 2} G_{1}(\eta, \rho)=\frac{e^{2 \pi \eta}-1}{2 \pi \eta} \\
& \quad \begin{aligned}
(4.7) \quad & \left\{\rho^{-1}+2 \eta[\log 2 \rho+\operatorname{Re} \psi(1+i \eta)+2 \gamma-3 / 2]\right\}\left(1+\eta^{2}\right)^{1 / 2} F_{1}(\eta, \rho) \\
& +\left(\rho^{-2}-2 \eta \rho^{-1}\right) F_{0}(\eta, \rho)-\left(1+\eta^{2}\right)^{1 / 2} D F_{1}(\eta, \rho) \\
& \left.\quad-4 \eta \sum_{L=1}^{\infty} \frac{2 L+1}{L(L+1)}(-1)^{L} \cos \delta_{0, L} D F_{L}(\eta, \rho)\right] .
\end{aligned}
\end{aligned}
$$

The latter result can be simplified as follows. The term $\rho^{-1} F_{1}(\eta, \rho)$ is eliminated by means of (4.5) with $L=1$. By properly combining (4.3) and (4.4) one has

$$
\begin{aligned}
D F_{L}(\eta, \rho)= & -\frac{\left(L^{2}+\eta^{2}\right)^{1 / 2}}{2 L+1} F_{L-1}(\eta, \rho)+\eta F_{L}(\eta, \rho) \\
& +\frac{\left[(L+1)^{2}+\eta^{2}\right]^{1 / 2}}{2 L+1} F_{L+1}(\eta, \rho)
\end{aligned}
$$

valid for $L=1,2, \cdots$. This result is substituted into (4.7). Then the infinite series in (4.7) can be rearranged using the identities

$$
\begin{aligned}
& \frac{\left[(L+1)^{2}+\eta^{2}\right]^{1 / 2}}{L(L+1)} \cos \delta_{0, L}-\frac{\eta(2 L+3)}{(L+1)(L+2)} \cos \delta_{0, L+1} \\
& -\frac{\left[(L+2)^{2}+\eta^{2}\right]^{1 / 2}}{(L+2)(L+3)} \cos \delta_{0, L+2} \\
& =\frac{2 L+3}{L(L+3)}\left[\sin \delta_{0, L+1}-\eta \cos \delta_{0, L+1}\right]=\frac{2 L+3}{L(L+3)}\left(1+\eta^{2}\right)^{1 / 2} \cos \delta_{1, L},
\end{aligned}
$$

(4.10) $\quad \cos \delta_{0,1}=-\frac{\eta}{\left(1+\eta^{2}\right)^{1 / 2}}, \quad \cos \delta_{0,2}=-\frac{2-\eta^{2}}{\left(1+\eta^{2}\right)^{1 / 2}\left(4+\eta^{2}\right)^{1 / 2}}$

which follow from (3.4) in a rather simple way. Omitting all further details, we present as our final expansion for $G_{1}(\eta, \rho)$,

$$
\begin{aligned}
& G_{1}(\eta, \rho)= \frac{e^{2 \pi \eta}-1}{2 \pi \eta}\left[\left\{\rho^{-2}-2 \eta \rho^{-1}+\frac{2}{3}\left(1+4 \eta^{2}\right)\right\} \frac{F_{0}(\eta, \rho)}{\left(1+\eta^{2}\right)^{1 / 2}}\right. \\
&+2 \eta\left\{\log 2 \rho+\operatorname{Re} \psi(1+i \eta)+2 \gamma-\frac{9}{4}-\frac{2}{3} \frac{1+4 \eta^{2}}{1+\eta^{2}}\right\} F_{1}(\eta, \rho) \\
&-\frac{1}{6}\left(4+\eta^{2}\right)^{1 / 2} F_{2}(\eta, \rho)-4 \eta \sum_{L=1}^{\infty} \frac{2 L+3}{L(L+3)} \\
&\left.\times(-1)^{L} \cos \delta_{1, L} F_{L+1}(\eta, \rho)\right] .
\end{aligned}
$$


The present result has been checked in a similar way as the expansion (4.2) for $G_{0}(\eta, \rho)$.

5. Computational Aspects. In practical applications of Coulomb wave functions, one often needs sequences of values $F_{L}(\eta, \rho), G_{L}(\eta, \rho)$ for fixed $\eta, \rho$ and $L=0$, $1, \cdots, L_{\max }$. The obvious tool for generating such sequences is the recurrence relation (4.5) which holds both for regular and irregular Coulomb wave functions. However, it is well known by now that the recursive computation of sequences may suffer from a serious loss of accuracy due to numerical instability.

In a recent paper [4] Gautschi investigates the problem of numerical instability for general three-term recurrence relations. In this connection he introduces the concepts of minimal solution and dominant solution of a recurrence relation. Starting from two initial values an application of the recurrence relation in the forward direction (i.e. in the direction of increasing order) yields a disastrous build-up of errors for the minimal solution, whereas the computation of the dominant solution remains numerically stable. Gautschi's paper mainly concentrates on the development of appropriate algorithms for the computation of minimal solutions, followed by applications to the computation of various transcendental functions. In [4, Section 7] the general theory is applied to the recurrence relation (4.5) for Coulomb wave functions. Owing to the behaviour of $F_{L}(\eta, \rho), G_{L}(\eta, \rho)$ as $L \rightarrow \infty$, viz.

$$
F_{L}(\eta, \rho) \sim C_{L}(\eta) \rho^{L+1}, \quad G_{L}(\eta, \rho) \sim \frac{\rho^{-L}}{(2 L+1) C_{L}(\eta)}, \quad L \rightarrow \infty
$$

with

$$
C_{L}(\eta)=\frac{2^{L} e^{-\pi \eta / 2}|\Gamma(L+1+i \eta)|}{(2 L+1) !}
$$

(cf. [1, Formula 14.6.3]), $F_{L}(\eta, \rho)$ is a minimal solution of $(4.5)$ and $G_{L}(\eta, \rho)$ is a dominant solution of (4.5). An algorithm for generating the set of values $F_{L}(\eta, \rho)$, $L=0,1, \cdots, L_{\max }$ is described. This algorithm uses the expansion (3.5) as its normalization identity. An ALGOL procedure based on this algorithm is described in [5].

A similar algorithm for the computation of regular Coulomb wave functions was proposed before by Stegun and Abramowitz [9], using the series (3.6) with $m=0$ for normalization. However, according to Gautschi [4] the latter algorithm becomes less accurate when $\eta$ and $\rho$ are positive and large.

The irregular Coulomb wave function being a dominant solution of (4.5), the sequence $G_{L}(\eta, \rho), L=0,1, \cdots, L_{\max }$ can be generated by means of the recurrence relation (4.5) applied in the forward direction. In order to start the recursion one first has to determine the initial values $G_{0}(\eta, \rho), G_{1}(\eta, \rho)$. The latter are readily obtained from the expansions (4.2), (4.11) substituting the set of values $F_{L}(\eta, \rho)$ as calculated before. Alternatively, one might also determine $G_{1}(\eta, \rho)$ from the Wronskian relation (cf. [1, Formula 14.2.5])

$$
F_{0}(\eta, \rho) G_{1}(\eta, \rho)-F_{1}(\eta, \rho) G_{0}(\eta, \rho)=1 /\left(1+\eta^{2}\right)^{1 / 2} .
$$

Finally we remark that the present scheme for the calculation of Coulomb wave functions shows a great similarity to an algorithm devised by Stegun and Abramowitz [10] for the computation of Bessel functions of the first and second kinds. The 
latter algorithm uses the expansion (2.13) for obtaining $Y_{0}(z)$ as compared to our scheme using the corresponding expansions (4.2), (4.11) for the computation of $G_{0}(\eta, \rho), G_{1}(\eta, \rho)$. As a matter of fact, it was this similarity which guided us in the present investigation.

Appendix. In this appendix we will evaluate the finite sums $S_{p}, T_{p}(a)$ as introduced in Section 2. It is shown that these sums can be expressed in terms of hypergeometric series with unit argument. The latter series can be summed explicitly by means of theorems of Gauss and Saalschütz, (cf. [3, Formulas 2.8(46), 4.4(3)]).

(i) Evaluation of $S_{p}$. In (2.7) $S_{p}$ was introduced as

$$
S_{p}=\sum_{r=0}^{p} \frac{(r+p+2 m) !}{(2 m+1)_{r}(p-r) ! r !}(-1)^{r} \frac{r+2 m-2 \kappa}{r+m-\kappa}
$$

which can be reduced to

$$
\begin{aligned}
S_{p}= & \frac{(p+2 m) !}{p !}\left\{{ }_{2} F_{1}(p+2 m+1,-p ; 2 m+1 ; 1)\right. \\
& \left.+{ }_{3} F_{2}\left[\begin{array}{c}
p+2 m+1, m-\kappa,-p ; 1 \\
2 m+1, m+1-\kappa
\end{array}\right]\right\} \\
= & (2 m) !\left[(-1)^{p}+\frac{(m+1+\kappa)_{p}}{(m+1-\kappa)_{p}}\right] .
\end{aligned}
$$

(ii) Evaluation of $T_{p}(a)$. According to (2.11) $T_{p}(a)$ is given by

$$
T_{p}(a)=\sum_{r=0}^{p} \frac{(r+p) !}{(r+1) !(p-r) ! r !}(-1)^{r} \psi(r+a) .
$$

The latter sum is meaningful provided $a \neq 0,-1,-2, \cdots$.

For $p=0$ it is obvious that

$$
T_{0}(a)=\psi(a) .
$$

For $p \geqq 1$ we substitute (cf. [3, Formula 1.7(10)])

$$
\psi(a+r)=\psi(a)+\sum_{j=0}^{r-1} \frac{1}{a+j} ;
$$

then (A.3) passes into

$$
\begin{aligned}
& T_{p}(a)= \psi(a) \sum_{r=0}^{p} \frac{(r+p) !}{(r+1) !(p-r) ! r !}(-1)^{r} \\
&+\sum_{r=1}^{p} \frac{(r+p) !}{(r+1) !(p-r) ! r !}(-1)^{r} \sum_{j=0}^{r-1} \frac{1}{a+j} \\
&=\psi(a)_{2} F_{1}(p+1,-p ; 2 ; 1) \\
&+\sum_{j=0}^{p-1} \frac{(j+p+1) !}{(j+2) !(p-j-1) !(j+1) !} \frac{(-1)^{j+1}}{a+j} \\
& \quad \times{ }_{3} F_{2}[j+p+2,1,-p+j+1 ; 1] . \\
& j+3, j+2
\end{aligned}
$$

These hypergeometric series can again be evaluated in the way mentioned above and one obtains 


$$
\begin{aligned}
T_{p}(a)= & \frac{1}{p(p+1)} \sum_{j=0}^{p-1} \frac{(j+p+1) !}{(j+1) !(p-j-1) ! j !} \frac{(-1)^{j+1}}{a+j} \\
= & -\frac{1}{p+1}\left\{{ }_{2} F_{1}(p+1,-p+1 ; 2 ; 1)\right. \\
& \left.\quad+\frac{p+1-a}{a}{ }_{3} F_{2}\left[\begin{array}{c}
p+1, a,-p+1 ; 1 \\
2, a+1
\end{array}\right]\right\} \\
= & \frac{1}{p(p+1)}\left[(-1)^{p}-\frac{(2-a)_{p}}{(a)_{p}}\right], \quad p=1,2,3, \cdots .
\end{aligned}
$$

Technological University

Department of Mathematics

Eindhoven, The Netherlands.

1. M. Abramowitz \& I. A. Stegun, (Editors), Handbook of Mathematical Functions with Formulas, Graphs and Mathematical Tables, Nat. Bur. Standards Appl. Math. Series, 55, Superintendent of Documents, U. S. Government Printing Office, Washington, D. C., 1964, 3rd printing with corrections, 1965. MR 29 \#4914; MR 31 \#1400.

2. H. BuchHOLz, Die konfluente hypergeometrische Funktion mit besonderer Berücksichtigung ihrer Anwendungen, Ergebnisse der angenwandten Mathematik, Bd. 2, Springer-Verlag, Berlin, 1953. MR 14, 978.

3. A. ERdÉLYI et al., Higher Transcendental Functions, Vols. I, II, McGraw-Hill, New York, 1953. MR 15, 419.

4. W. GAUTSCHI, "Computational aspects of three-term recurrence relations," SIAM Rev., v. 9, 1967, pp. 24-82. MR 35 \#3927.

5. W. GauTsCHI, "Algorithm 292-regular Coulomb wave functions," Comm. ACM, v. 9, 1966, pp. 793-795.

6. A. Messiah, Mécanique Quantique, Vol. I, Dunod, Paris, 1959; English transl., NorthHolland, Amsterdam and Interscience, New York, 1961. MR 23 \#B2340. MR 23 \#B2826.

7. Nat. Bur. Standards, Tables of Coulomb Wave Functions. Vol. I, Appl. Math. Series, no. 17, U. S. Government Printing Office, Washington, D. C., 1952. MR 13, 988.

8. L. J. Slater, Confluent Hypergeometric Functions, Cambridge Univ. Press, New York, 1960. MR $21 \# 5753$.

9. I. A. Stegun \& M. Abramowitz, "Generation of Coulomb wave functions by means of recurrence relations," Phys. Rev., v. 98, 1955, pp. 1851-1852. MR 16, 1155.

10. I. A. Stegun \& M. Abramowitz, "Generation of Bessel functions on high speed computers," Math. Comp., v. 11, 1955, pp. 255-257. MR $20 \# 459$.

11. G. N. Watson, A Treatise on the Theory of Bessel Functions, 2nd ed., Cambridge Univ. Press, Cambridge and Macmillan, New York, 1944. MR 6, 64. 